\title{
'One Health' infectious diseases surveillance in Tanzania: Are we all on board the same flight?
}

\begin{abstract}
Authors:
Erasto V. Mbugi ${ }^{1,2}$

Kim A. Kayunze ${ }^{3,4}$

Bugwesa Z. Katale

Sharon Kendall ${ }^{6}$

Liam Good ${ }^{6}$

Gibson S. Kibik ${ }^{7}$

Julius D. Keyyu ${ }^{5}$

Peter Godfrey-Faussett ${ }^{8}$

Paul van Helden ${ }^{9}$

Mecky I. Matee ${ }^{1}$

\section{Affiliations:}

${ }^{1}$ Department of Microbiology and Immunology, Muhimbili University of Health and

Allied Sciences, Tanzania

${ }^{2}$ Department of Biochemistry, Muhimbili University of Health and Allied Sciences, Tanzania

${ }^{3}$ School of Public Health and Social Sciences, Muhimbili University of Health and Allied Sciences, Tanzania

${ }^{4}$ Development Studies Institute, Sokoine University of Agriculture, Morogoro

${ }^{5}$ Tanzania Wildlife Research Institute, Arusha, Tanzania

${ }^{6}$ The Royal Veterinary College, London, United Kingdom
\end{abstract}

${ }^{7}$ Kilimanjaro Clinical Research Institute, Tumaini University, Tanzania

${ }^{8}$ London School of Hygiene and Tropical Medicine, London, United Kingdom

${ }^{9}$ DST/NRF Centre of Excellence for Biomedical Tuberculosis Research/MRC Centre of Molecular and Cellular Biology, Division of Molecular Biology and Human Genetics, Faculty of Health Sciences, University of Stellenbosch,

South Africa

Correspondence to:

Erasto Mbugi

Email:

erasto.mbugi@sacids.org

Postal address:

PO Box 65001, Dar es

Salaam, Tanzania
Infectious diseases account for nearly $40 \%$ of the burden of human mortality and morbidity in low-income countries, of which $7 \%$ is attributable to zoonoses and $13 \%$ to recently emerging diseases from animals. One of the strategic approaches for effective surveillance, monitoring and control of infectious diseases compromising health in both humans and animals could be through a combination of multiple disciplines. The approach can be achieved through a joint effort from stakeholders comprising health professionals (medical and veterinary), social, economic, agricultural, environmental and other interested parties. With resource scarcity in terms of number of staff, skills and facility in low-income countries, participatory multisectoral and multidisciplinary approaches in limiting the burden of zoonotic diseases could be worthwhile. We review challenging issues that may limit the 'One Health' approach for infectious diseases surveillance in Tanzania with a focus on Health Policy and how best the human and animal health systems could be complemented or linked to suit the community in need for disease control under the theme's context.

\section{Introduction}

The concept of 'One Health', if deployed in resource poor countries with proper management may be the best approach in sustainable control of emerging and re-emerging zoonoses in these countries. A recent report (Jones et al. 2011) suggests that infectious diseases account for nearly $40 \%$ of the human disease burden with respect to morbidity and mortality in low-income countries. One-fifth of this is attributable to zoonoses or diseases which are classified as recently emerged from animals (7\% and 13\% respectively). The 1st 'One Health' International Congress was held in Melbourne (from 14 February to 16 February 2011) with substantial representation from the Southern African Centre for Infectious Diseases Surveillance (SACIDS), senior and young researchers from around the world as well as senior representatives from United Nations (UN), Food and Agriculture Organisation of the United Nations (FAO), World Health Organisation (WHO) and Office International des Epizooties (OIE). The meeting addressed, amongst many issues, the 'One Health' concept of infection and immunity and the concept was illustrated by discussion about influenza virus which was first isolated from pigs in 1931 as a probable wildlife reservoir (Doherty 2011). The connection and suspicion of connection to an avian and porcine reservoir of recent influenza outbreaks, originating usually in Asia, is widely known (Taubenberger \& Morens 2006) and it is likely that the 1918 'flu epidemic had a similar origin (Morse 2004). One of the major issues in control of infections transmissible to man and animals is the lack of or limited joint approach to ameliorate the situation. Thus the need for joint action with a combination of technologies and conceptual frameworks from both medical and veterinary professionals, social scientists and ecologists to launch approaches to contain the emerging and re-emerging infectious diseases. Again, a reference to the Asian situation is useful: in this scenario, we may expect regular emergence of influenza epidemics, which cycle between poultry and pigs kept in close contact, and where the virus gains virulence in this cycling, enabling it to become highly virulent to humans in due course. The need for effective integration between human and animal surveillance has been repeatedly identified as a key to successful surveillance of emerging and re-emerging infectious diseases (Cleaveland, Laurenson \& Taylor 2001; Kahn 2006; Kuiken et al. 2005; Morse 1995; Murphy 1998; Shears 2000; Woolhouse 2002; Woolhouse \& Gowtage-Sequeria 2005; World Bank 2010).

Nevertheless, there are challenges to the 'One Health' concept, particularly regarding its operationalisation. Early detection and surveillance of the warning signs of infectious diseases is

How to cite this proceeding: Mbugi, E.V., Kayunze, K.A., Katale, B.Z., Kendall, S., Good, L., Kibik, G.S. et al. 2012, "'One Health" infectious diseases surveillance in Tanzania: Are we all on board the same flight?', Onderstepoort Journal of Veterinary Research 79(2), Art. \#500, 7 pages. http://dx.doi.org/10.4102/ojvr.v79i2.500

Note: Proceedings of the Conference of the Southern African Centre for Infectious Disease Surveillance 'One Health' held at the National Institute for Communicable Diseases, Johannesburg, July 2011. This article was republished with the author's full affiliation.

Copyright: @ 2012. The Authors. Licensee: AOSIS OpenJournals. This work is licensed under the Creative Commons Attribution License. 
one major key towards successful control of diseases, in both humans and animals, but the lack of participatory health policy that focuses on multi-sectoral contributions towards One Health is a major hurdle, particularly in resource-poor countries. This means that having an interdisciplinary consensus commitment and collective networks with leading consortia such as the SACIDS, One Health Central and East Africa (OHCEA) and United States Agency for International Development (USAID) organisation for prevention of disease outbreaks in central Africa (RESPOND) as prominent networks could initiate 'One Health' action by breaking down barriers and creating networks. These could contribute to alleviating cross-transmission of infections at the human/ animal/ecosystem interface which is a critical step to limit interspecies disease transmission. The approach could exploit skills from various disciplines, to prevent emerging and re-emerging infectious diseases from becoming prevalent, probably with minimal costs in terms of human and other resources. In this context, the major focus could be on social and cultural determinants of health that necessitate a holistic, integrative and interdisciplinary approach to alleviate health problems. The Animal Health for the Environment and Development (AHEAD) grouping in South Africa, which is also affiliated with the Wildlife Conservation Society (WCS) is an example of organisations applying One Health approaches in disease surveillance and control.

Zoonotic infections from both livestock and wildlife in some countries contribute to infections in man for example, $60 \%$ zoonoses of which $70 \%$ originate from wildlife (Atlas 2011; WHO 2011). It is clear that the risk of disease outbreaks is associated with climate, land use, scarce resources, innate ecosystem characteristics as well as poverty (Daszak 2011). There is growing demand for meat and animal products, which is likely to lead to increasingly intensive farming and in turn and increased risk for disease. International Livestock Research Institute (ILRI) (Kenya) reports that 'a new infectious disease emerges every four months, and 75\% of them originate in animals', according to a recent report in Nature. Gilbert (2011) stated:

Such emergent diseases can have severe socio-economic, health and environmental impacts. Whereas rich nations are controlling livestock diseases effectively, developing countries, including many in Africa and Asia, lag dangerously behind. (Gilbert 2011)

Cook et al. (1996) reported an association between human tubercle bacillus (TB) cases and tuberculin positive cattle and clearly indicated the need for both medical and veterinary authorities' joint action to arrest infection and subsequent disease. Yet there have been few studies on risk factors for transmission from wild or domestic animals to people and between wildlife and domestic livestock in the literature (Jones et al. 2011). Therefore, the effect of One Health action could be more than just an attempt to limit zoonotic infectious diseases, since it will extend to food security. It has been predicted that lack of food security is an increasing and serious risk to life, the lack of which is likely to result in more deaths in some areas than the combination of malaria and HIV and AIDS (Nabarro 2011). It has been reported that up to $40 \%$ of household income in developing countries can be livestock dependent (McDermott, director of ILRI, and his ILRI colleague Delia Grace at a conference in New Delhi [Leveraging agriculture for improving nutrition and health]). Therefore, all these health related issues need attention and by addressing them together and holistically is likely to be worthwhile. The only question is whether all disciplines, including the broad definitions of natural and social sciences, are ready to be integrated. Environment, for example, can play role in disease transmission to both humans and animals. Evidence exists from some parts of the world for contamination of meat with Clostridium difficile (Riley 2011) raising concern on human and animal health through cross contamination.

For example, preventing the spread of some infections from wildlife (e.g. Ebola fever), can be achieved by effecting behavioural changes in hunters, but this is perhaps best done by professionals other than medical or veterinary workers, since it requires a good understanding of educational approaches at the appropriate level to hunters concerning wildlife health. An associated benefit of such intervention would enable the hunters to participate in wildlife health monitoring systems (Reed \& Cameron 2011; Ondzie et al. 2011). The issue of social mobilisation is vital in One Health so there needs to be a strong emphasis on the need for national, regional and international collaboration in disease control (Odugleh-Kolev, WHO offices, Geneva, Switzerland). Diseases have extensive social impact, and high economic costs, thus the need for a participatory and a well-coordinated approach, which requires the full multidisciplinary approach, including economists and politicians.

There are several drivers of disease emergence and reemergence, and we know that evolution of microorganisms involve a dynamic relationship with their hosts and environment (Morens, Folkers \& Fausi 2004). Therefore, smart surveillance, using for example phylogenetics, can reveal the source of disease and direction of flow. Such information can provide critical information to enable the problems to be tackled by the appropriate professionals with the correct tools in the practice of the 'One Health' approach.

\section{Health policy systems in Tanzania}

Despite advocating 'One Health' approaches in infectious disease surveillance in developing countries such as Tanzania, the concept may be challenged and compromised by the existing health policies in the country. The current health policy and the Tanzanian Veterinary Act (the tool that provides guidelines for veterinary practice activities) may not have a common point of intersection. This could be a result of parallel working organs that do not interact or a habitually conservative notion that 'a vet should be a vet' and 'a medic should be a medic'. This concept could also be applicable to other professionals, whose roles could be critical if the 'One Health' concept should be attempted. The concept of 'One Health' can be misunderstood and needs to be introduced carefully; for example, one could think that with the concept, 
a vet can work in a medical hospital, and similarly a medical practitioner in a vet clinic. This is not the idea under 'One Health', since the goal is to attempt to utilise professional skills from both veterinarians and medical practitioners as well as other professionals in arresting zoonotic diseases. The differential diagnosis of zoonotic diseases in humans may require the skills of both health professionals, followed up with preparation of intervention plans enabling the optimal use of veterinary intervention as a means to reduce the burden of human disease from an animal source (Michel, Müller \& Van Helden 2010). The idea is to create preparedness in disease control through early disease surveillance, which is a critical initial step in restraining spread of diseases in developing countries. A study by Karimuribo et al. (2011) points out lack of formal routine collaboration between sectors in disease surveillance with cross-sector collaboration being triggered only following disease emergencies. This has resulted in poor success in controlling disease outbreaks because of the lack of joint preparation for epidemics. Examples include diseases such as Avian influenza, Rift Valley fever (RVF) and African Swine fever. The zoonotic potential of many diseases is not even recognised by many animal care practitioners, let alone medical practitioners (Swai, Schoonman \& Darbon 2010).

The emergence of novel diseases across the animal and human domains is said to result from the rapid increase of both human and domestic animal populations. This increase has consequently increased the contact rate (Jones et al. 2011; Mazet et al. 2011; Michel et al. 2006). This fact necessitates the need for a change in strategy for disease control, shifting from a single to a multidisciplinary approach. Countryspecific health systems can largely contribute to limiting efforts for 'One Health' despite any good will for successful strategic disease control. Available reports (Department for International Development 2011) have in principle indicated the public health burden of zoonotic tuberculosis in Tanzania. For example, this report identifies Mycobacterium bovis, Mycobacterium tuberculosis and atypical mycobacterial species isolated from biopsy samples from human patients to be the main causes of tuberculous adenitis in humans. Therefore the report highlights the need for a veterinarymedical collaborative effort to control tuberculosis in Tanzania. Collaborative efforts between these health professional partners have been improving over time but are largely dependent on the respective policies that govern the two bodies independently. Not only the policies, but also a change in mindset of the individual practitioners from the two but closely related fields to a holistic approach in the control of zoonoses is needed for adoption of One Health. Clearly, a major limiting factor for both professionals is the extremely limited resource base, shortage of qualified personnel leading to a lack of time to deal with routine daily problems and the huge burden of disease. There is thus insufficient time or inducement to reflect and plan to move to a common ground.

The Tanzanian health policy currently emphasises the need for planning health development since resources and technology are limited (Ministry of Health of Tanzania 2003). The policy also recognises the role of traditional and alternative health care to Tanzanians, which is a significant component of the health care since about $60 \%$ of the population use traditional and alternative care systems for their day-to-day health care. However, it does not indicate the need for joint efforts between the veterinary, medical and other professionals as players which would provide for the establishment of 'One Health'. The Tanzanian Ministry of Health and Social Welfare (Ministry of Health and social welfare of Tanzania 2009) stresses the value of Partnership for Delivering the Millennium Development Goals (MDGs). In its report, it recognises the need for preparedness for emerging and re-emerging diseases due to intensive crossborder contacts and globalisation. It is amongst the strategic plans for the Ministry of Health and social welfare of Tanzania (MOHSW) to strengthen surveillance, prevention, diagnosis and treatment of neglected infectious tropical diseases. However, the strategic plan does not clearly indicate the role of other professionals who could be instrumental for early diagnosis and prevention of infections particularly zoonoses. The concept of effective integration of the medical and veterinary disciplines has been suggested to be particularly necessary in the low income countries where facilities are limited (Shears 2000). Perhaps the time has come to address this in formulating a new MOHSW plan in collaboration with the other relevant ministries, for example, Ministries of: Agriculture, Food Security and Co-operatives; Livestock and Fisheries Development; Water and Irrigation and Natural Resources and Tourism.

\section{Zoonoses with reference to previous veterinary studies}

As far as veterinary and medical practices are concerned, zoonoses are not part of routine differential diagnosis in Tanzania. This has, in addition, not been part of advisory packages to livestock keepers (John, Kazwala \& Mfinanga 2008; Swai et al. 2010). This lack of attention to otherwise preventable diseases has consequently resulted in underreporting and lack of proper treatment to patients and livestock owners in need. A study by Swai et al. (2010) on the knowledge and attitude towards zoonoses in both animal health workers and livestock keepers proposed, based on their findings, the need for public health promotion and education using an inter-disciplinary one-health collaboration between vets, public health practitioners and policy makers. It was further suggested that there should be joint action amongst these stakeholders and that they are the key to a more efficient and effective approach to the diagnosis and control of zoonoses in Tanzania. The current inadequate communication between veterinary and human health care professionals in approaching these issues plus the limited data on zoonoses in Tanzania (Cripps 2000) is a factor constraining timely diagnosis and control of these diseases.

\section{Climatic change and One Health}

Climate change influences the pathogen flow, disease transmission and spread to various hosts. In a similar 
context, climate change is predicted to affect the geographical distribution and population densities of wildlife, which consequently will affect disease dynamics (Jones et al. 2011). Strong evidence suggests pathogen flow between people, livestock and non-human primates; and predicts that flow will increase concomitant with forest fragmentation and edge effects (Jones et al. 2011). On the other hand, agro-ecosystem change has been said to decrease disease risk and flow from wildlife to domestic animals whilstalso selecting for decreased pathogenicity (Jones et al. 2011). In its strategic plans, the Tanzanian Ministry of Health highlights those threats that may rapidly prove to be destructive, for example Avian Flu, Severe acute respiratory syndrome (SARS) and other new threats due to climate change (Ministry of Health and social welfare of Tanzania 2009). The One Health approach should therefore acknowledge and attempt to incorporate complex socioecological dynamics that influence the health of humans and animals (both livestock and wildlife). Clearly, this will not be possible without the inclusion of social and natural scientists in the One Health plan

The OHCEA, which is another network for infectious disease surveillance and control, has objectives that mainly focus on emerging diseases for sustainable health and the need for a transformational change that allows continuous improvement of health and well-being of humans, livestock as well as wildlife ecosystems. The main goal, like other 'One Health' based networks, focuses on a holistic approach to improve public and animal health. Linked 'One Health' networks will make information sharing within and between vicinities easier in terms of skills and approach. In a similar context, the success for a 'One Health' approach in disease surveillance and control rests on acceptance and active participation of both national and international multidisciplinary key partners.

Available evidence suggests that socio-economic determinants of health are more powerful determinants of risk than biological factors (Jones et al. 2011). Therefore, the need for biocultural and economic approaches for 'One Health' operationalisation is necessary. The major challenge in these health approach issues is the inequalities within and between human populations that produce different levels of disease burden in human populations. Attention to food safety and security, particularly livestock products from sub-Saharan Africa and a comprehensive, focused resultbased collaborative approach whilst taking responsibility towards 'One Health' is a key towards success. Therefore, people should address the 'One Health' concept at national and international levels with special focus on possible identification of points of intervention, feasibility and acceptability of the approach by the community in need. This is possible through a joint approach amongst disciplines in disease control strategies.

\section{Drug resistance}

Antimicrobial drug resistances as well as possible crossresistances are other major challenges to the One Health approach in surveillance and control of diseases. In human tuberculosis, for instance, drug resistance as well as multiple drug and extensively multi-drug resistance (MDR and XDR$\mathrm{TB})$ pose a challenge to the treatment of human tuberculosis. It is the strategy of the Tanzanian Ministry of Health and Social Welfare to introduce and implement multi-drug and extensively multi-drug resistance TB management (Ministry of Health and social welfare of Tanzania 2009). However, the strategic plan does not cite specific key role players, nor highlight the risk of treating $M$. bovis infection in humans with standard first line drugs without careful follow-up, given that $M$. bovis is innately resistant to pyrazinamide. Given this, there is a very high risk that multi-drug resistant $M$. bovis could become a reality and may flow back to an animal reservoir over time. However, if veterinarians and other professionals are given opportunities where appropriate, the outcome should be more favourable. The advantage is to arrest the situation early and at relatively low cost whilst sharing the available scarce resources in order to avoid high or unaffordable costs later.

Successful operationalisation of the concept of 'One Health' can be achieved however, it needs a careful assessment of areas of weakness as far as health policies are concerned (Delegates at the 1st One Health Conference, Melbourne, February 2011). Amongst these we need to highlight the possible economic impacts of emerging infectious diseases, thus the need for political and policy actions to rectify the situation. These all are achievable through improved sentinel surveillance systems and better laboratory and multidisciplinary epidemiological research.

The 'One Health' concept targets early detection of disease at the animal or human and ecosystem interface to fulfill its mandate. This is not a straightforward approach, as it touches on multi-sectoral policies, consensus and community education. In most communities, animals are deeply interwoven with the social system and are used as 'savings' amongst the rural people (Michel et al. 2004), making it difficult to enforce culling even when animals are clearly infected with a disease. This is particularly problematic if there is little or no compensation as is the case in Tanzania. Therefore, communities as stakeholders need to be willing participants to the processes. Education to the community will definitely aid in avoiding misunderstanding of the concept of 'One Health' amongst people of different calibre. A concern regarding the 'One Health' approach to disease surveillance is the possibility of misunderstanding the concept itself. It should be understood that the aim of the concept is to increase preparedness for tackling infectious diseases, special attention being given to the emerging unexpected and novel events with Avian flu, influenza, RVF and SARS as few examples.

\section{Effective change}

Awareness is the key to eventual change from one system to another. Dedicated efforts are needed to ensure education of stakeholders in 'One Health', particularly increasing 
awareness targeting specific groups such as farmers and lay people who might have no knowledge or awareness of the concept. A broader perspective on zoonoses should be deployed, particularly to change the local knowledge in both traditional and smallholder livestock keeping systems which might be limited to a restricted number of zoonoses such as anthrax, rabies and tuberculosis (Swai et al. 2010). Attention to large scale commercial farming is also important. Effective positive changes under the theme 'One Health' require involvement and engagement at the higher hierarchal level of leadership, using compelling arguments regarding environment, sustainability, lives at risk, economic impact, security as well as instability. This will hopefully lead to appropriate action at policy level, which will include the setting of balanced and reasonable priorities. The idea is to have a cross-agency and cross-disciplinary collaboration in science and humanities and bring together the key policy players whose role in 'One Health' is crucial. The greatest challenge to this approach is the presence of outdated policies in different government sectors for disease control that might require transformation in terms of thinking amongst these key players into practical and action-oriented plans. A clear definition of 'One Health' has to be spelled out. The provisionally suggested definition is that 'One Health is the prevention of human disease through control of animal infections and diseases in all ecosystems (Stakeholders at the 1st One Health Conference, Melbourne, Australia, February 2011). To achieve this goal, having a cadre of dedicated and integrated medical and veterinary professionals as well as other key players including sociologists, policy makers and the politicians should be a priority. This holistic approach in control of zoonoses could be rewarding particularly in third world countries where the cost of disease (not only in economic terms) is high and there are limited human and other resources.
Thebarriers and bridges for an inter-professional participatory approach in zoonotic disease control have been identified and critically analysed (World Health Organisation/Department for International Development Animal Health Program, Food and Agriculture Organisation \& Office International des Epizooties 2006). These factors have been clustered into bridges and barriers (Table 1), with barriers outnumbering bridges and have been proposed to be useful checklists to guide planning, organisation and management of zoonosis research in future. The idea is to allow veterinary and medical authorities to identify and tackle some of the overarching problems such as the lack of emphasis on zoonotic diseases in the training of both medics and vets. This is critical if we want to control zoonotic diseases under a 'One Health' approach, as it may be influenced by a bureaucratic modus operandi in decision-making, resulting from the lack of welldefined policies for inter-sectoral linkage as a disease control strategy.

\section{One Health and Bureaucratic Organisation}

The 'One Health' approach provides the opportunity for joint global health initiatives with the opportunity to use resources optimally compared to use in one sector only. With this approach it is a good idea to identify cross organisational boundaries with the aim of reducing redundancy, whilst increasing efficiency and improving outcomes for people, animals, the environment and society (comments from Joseph Anelli, a stakeholder in 'One Health' at the 1st One Health Conference, Melbourne, February 2011). Various pandemics have in principle indicated the impact that emerging infectious diseases can have economically, socially, medically, and environmentally, suggesting benefits of using a 'One Health' approach in education to address global health and sustainability challenges (Barret et al. 2010). This

TABLE 1: Bridges and barriers in controlling zoonotic diseases. Bridges
Collaboration, integration, networking and partnership from inception to completion of
both research and control activities and bringing together both medics and vets and basic and applied research

Buy-in from professional associations

Sufficient money in budget or budgets

Capacity building: common training in zoonotic diseases for both veterinary and medical doctors and fieldworkers

Dual benefit: gains for animal and human health

Demand-driven, problem-led research

\section{Barriers}

Rivalry (institutional and professional), competition, institutional separation, poor linkages (e.g. between ministry of health and veterinary authorities)

Bureaucratic bottlenecks: who makes the decisions?

Control of zoonotic diseases based on fire-fighting or crisis management

Unregulated international trade in livestock and livestock products hampers control

Lack of clarity about roles of the public and private sector partners

Confusion: who pays, who gains?

Lack of resources

Budgetary separation: veterinary and medical costs not pooled

Training: lack of emphasis on zoonotic diseases

Weak veterinary public health infrastructure

Difference of emphasis: medics focus on individual patients, vets on populations

Malaria: cattle can be important in its epidemiology, yet it is not a zoonosis so is not included in zoonotic initiatives

Research being not demand-driven

but donor-led

Applied research is not recognized or rewarded as being as important as basic research Inadequate resources for dissemination of results and raising public awareness Lack of consensus on priority-setting 
approach could provide added value through combined efforts, rather than integration of the veterinary profession into the medical profession for example, which is neither a desirable outcome nor the intention. It is quite clear that the idea of 'One Health' cannot be overemphasised; it is 'predict' and 'prevent' rather than 'cure' and 'treat' (Simon Easteal, stakeholder in 'One Health' at The 1st One Health Conference, Melbourne, February 2011). This means that for a successful One Health approach, greater understanding of biological systems including the molecular mechanisms is needed. In addition, we need a better understanding of the epidemiology of infectious diseases at the human and animal (livestock and wildlife) intersection. This is achievable through education and training to achieve knowledge and skills gain amongst different health professionals. In addition, it would be important to raise awareness by facilitating communication and inter-disciplinary collaboration on research, information sharing amongst veterinary, public health, agricultural personnel and policy makers (Coulibaly \& Yameogo 2000). The need to understand the dynamics of infectious diseases in their complex multi-host communities, their threats to public health, livestock economies and wildlife has been spelled out earlier (Cleavaland et al. 2001). The simplest way to achieve this at ground level at minimal cost is to organise joint meetings and symposia with different professionals to address a common disease problem, for example, avian influenza. Using such an example, one can have professionals such as vets, clinicians, conservation specialists in birds and bird movement and animal and laboratory technicians. It is the systematic quantification of these cross-species transmitted pathogens that need a joint approach amongst stakeholders to restrain their impacts that could be viewed in public health, conservation and economic perspectives. These all efforts will be successful with policies that allow multi-sectoral approach in disease control, particularly those of interest to both animals and humans.

\section{Conclusion}

Zoonotic diseases are arguably more important in low-income countries than in high-income resource-rich countries. The emergence and re-emergence of zoonotic diseases in lowincome countries is due to a number of factors including deficient policies, population growth, environment and ecosystem change and a misconception of the 'One Health' concept amongst professionals. In addition, these factors are compounded by a low level of preparedness. Finally, there is the need to work towards acceptance by communities and authorities in need for a change in strategies to control zoonotic infections. This necessitates the need for effective understanding of 'One Health' and associated networks to communicate and generate a shared database of 'One Health' perspectives for effective control of both commonly known and emerging zoonoses. Perhaps the most relevant route to identify the optimal points of intervention should be through national and international collaboration and coordination using available resources in terms of personal skills and technology for disease surveillance, diagnosis, treatment and control. The change in policy to favour a holistic approach and interaction in disease surveillance and control could be a remarkable boost to environmental preservation, conservation strategies, as well as human and animal health and economic well-being.

\section{Acknowledgement}

The SACIDS is acknowledged for providing the opportunity for a Postdoctoral Research Fellowship to EM. Financial support was received from the Wellcome Trust subawardees grant. Prof. Mark Rweyemamu, the Director SACIDS is acknowledged for stimulating the idea and advice. The London International Development Centre in the United Kingdom and its staff is acknowledged for collaboration and providing the opportunity for preparation of this manuscript. Prof. Jeff Waage is acknowledged for his dedicated efforts to provide complementary material support and encouragement during preparation of this manuscript. Muhimbili University of Health and Allied Science, particularly, the School of Medicine and The Department of Biochemistry are acknowledged for granting time for preparation of this work.

\section{Competing interests}

The authors declare that they have no financial or personal relationship(s) which may have inappropriately influenced them in writing this paper.

\section{Authors' contributions}

E.V.M. (Muhimbili University of Health and Allied Sciences) participated in reviewing the literature, conceptualising, drafting and writing the manuscript. B.Z.K. (Tanzania Wildlife Research Institute) participated in initial drafting of the manuscript and critical manuscript review. S.K. (The Royal Veterinary College) participated in critical manuscript review. L.G. (The Royal Veterinary College) assisted in critical manuscript review and focusing of the manuscript. G.S.K. (Kilimanjaro Clinical Research Institute) critically reviewed the manuscript. J.D.K. (Tanzania Wildlife Research Institute) conceived the idea, assisted in drafting and critical review of the manuscript. P.v.H. (University of Stellenbosch) assisted in critical review of the manuscript. P.G.F. (London School of Hygiene and Tropical Medicine) assisted in drafting the manuscript and critically reviewed the manuscript. M.I.M. (Muhimbili University of Health and Allied Sciences) directed the whole process of drafting and critical review of manuscript. K.A.K. (Muhimbili University of Health and Allied Sciences) participated in initially reviewing the first draft of the manuscript and in critical subsequent manuscript review.

\section{References}

Atlas, R., 2011, 'Emerging infectious diseases: Rethinking zoonoses', First Internationa One Health Congress Abstracts, EcoHealth 7, S8-S170. http://dx.doi.org/10.1007/ s10393-010-0376-0

Cleaveland, S., Laurenson, M.K. \& Taylor, L.H., 2001, 'Diseases of humans and their domestic mammals: Pathogen characteristics, host range and the risk of emergence', Philosophical Transactions of the Royal Society of London, Series B: Biological Sciences 356, 991-999. http://dx.doi.org/10.1098/rstb.2001.0889, PMid:11516377 
Cook, A.J.C., Tuchili, L.M., Buve, A., Fosterm, S.D., Godfrey-Faussett, P., Pandey, G.S et al., 1996, 'Human and bovine tuberculosis in the Monze District of Zambia-A et al., 1996, 'Human and bovine tuberculosis in the Monze Di
cross-sectional study', British Veterinary Journal 152(1), 37-46.

Coulibaly, N.D. \& Yameogo, K.R., 2000, 'Prevalence and control of zoonotic diseases: Collaboration between public health workers and veterinarians in Burkina Faso', Acta Tropica 76, 53-57. http://dx.doi.org/10.1016/S0001-706X(00)00090-5

Cripps, P.J., 2000, 'Veterinary education, zoonoses and public health: A personal perspective', Acta Tropica 76, 77-80. http://dx.doi.org/10.1016/S0001706X(00)00094-2

Daszak, P., 2011, 'Smart surveillance: Analyzing environmental drivers of emergence to predict and prevent pandemics', First International One Health Congress Abstracts, EcoHealth 7, S8-S170.

Department for International Development report, 2011, 'Mycobacterium bovis infection of cattle and man in Tanzania', in R4D Project, viewed 19 May 2011, from http://www.dfid.gov.uk/r4d/SearchResearchDatabase. asp?ProjectID=2048

Doherty, P., 2011, 'The one-world of infection and immunity', First International One Health Congress Abstracts, EcoHealth 7, S8-S170. http://dx.doi.org/10.1007/ s10393-010-0376-0

Gilbert, N., 2011, 'Livestock plagues are spreading', Nature. http://dx.doi. org/10.1038/news.2011.87

Grace, D., Pfeiffer, D., Kock, R., Mutua, F. \& McDermott, J., 2011, 'A systematic review of zoonoses transmission and livestock/wildlife interactions', First International One Health Congress Abstracts, EcoHealth 7, S8-S170. http://dx.doi.org/10.1007/ s10393-010-0376-0

John, K., Kazwala, R. \& Mfinanga, G.S., 2008, 'Knowledge of causes, clinical features and diagnosis of common zoonoses among medical practitioners in Tanzania', BMC Infectious Diseases 2, 162. http://dx.doi.org/10.1186/1471-2334-8-162, PMid:19046464

Jones, B., McKeever, D., Grace, D., Pfeiffer, D., Mutua, F., Njuki, J. et al., 2011 'Zoonoses (Project 1) Wildlife/domestic livestock interactions', A draft report to the Department for International Development (DFID), UK; Submitted by: The International Livestock Research Institute, Nairobi \& Royal Veterinary College, London. 20th April, 2011 (DRAFT 4).

Kahn, L.H., 2006, 'Confronting zoonoses, linking human and veterinary medicine', Emerging Infectious Diseases 12, 556-561. http://dx.doi.org/10.3201/ eid1204.050956, PMid:16704801

Karimuribo, E., Wambura, P., Mounier-Jack, S., Sonoiya, S., Short, N., Aanensen, D. et al., 2011, 'Contrasting features and opportunities for "One Health" infectious diseases surveillance system in Tanzania', First International One Health Congress Abstracts, EcoHealth 7, S8-S170. http://dx.doi.org/10.1007/s10393-010-0376-0

Kuiken, T., Leighton, F.A., Fouchier, R.A.M., LeDuc, J.W., Peiris, J.S.M., Schudel, A. et al., 2005, 'Public health: Pathogen surveillance in animals', Science 309, 1680-1681. $\mathrm{http}: / /$ dx.doi.org/10.1126/science.1113310, PMid:16150997

Mazet, J.A.K., Clifford, D.L., Coppolillo, P.B., Deolalikar, A.B., Erickson, J.D. \& Kazwala, R.R., 2011, 'A “One Health" approach to address emerging zoonoses: The HALI
project in Tanzania', PLoS Med 6, e1000190. http://dx.doi.org/10.1371/journal. project in Tanzania', PLoS Med
pmed.1000190, PMid:20016689

Michel, A.L., Bengis, R.G., Keet, D.F., Hofmeyr, M., De Klerk, L-M., Cross, P.C. et al., 2006, 'Wildlife tuberculosis in South African conservation areas: Implications and challenges', Veterinary Microbiology 112, 91-100. http://dx.doi.org/10.1016/j. challenges', Veterinary Microbiology
vetmic.2005.11.035, PMid:16343819

Michel, A.L., Meyer, S., McCrindle, C.M. \& Veary, C.M., 2004, 'Community based veterinary public health systems, current situation, future trends and recommendations', in FAO Expert Consultation on Community Based Veterinary recommendations, in FAO Expert Consultation on Community Based Veterinary programmes/en/vph/events/expert_consult_reportpdf.

Michel, A.L., Müller, B. \& Van Helden, P.D., 2010, 'Mycobacterium bovis at the animalhuman interface: A problem, or not?', Veterinary Microbiology 140, 371-381. http://dx.doi.org/10.1016/j.vetmic.2009.08.029, PMid:19773134
Ministry of Health and social welfare of Tanzania, 2009, Health Sector Strategic Plan III: 'Partnership for Delivering the MDGs', Ministry of Health and Social Welfare, The United Republic of Tanzania.

Ministry of Health of Tanzania, 2003, The Tanzania National Health Policy, Ministry of Health and Social Welfare, The United Republic of Tanzania.

Morens, D.M., Folkers, G.K. \& Fausi, A.S., 2004, 'The challenge of emerging and reemerging infectious disease', Nature 430, 242-249. http://dx.doi.org/10.1038/ nature02759, PMid:15241422

Morse, S.S., 2004, 'Factors and determinants of disease emergence', Revue Scientifique et Technique 23, 443-451. PMid:15702712

Morse, S.S., 1995, 'Factors in the emergence of infectious diseases', Emerging Infectious Diseases 1, 7-15. http://dx.doi.org/10.3201/eid0101.950102, PMid:8903148

Murphy, F.A., 1998, 'Emerging zoonoses', Emerging Infectious Diseases 4, 429-435. http://dx.doi.org/10.3201/eid0403.980324, PMid:9716965

Nabarro, D., 2011, 'The UN system High Level Task Force (HLTF) on the Global Food Security Crisis and the Updated Comprehensive Framework for Action (UCFA)', 1st International One Health Congress Abstracts, EcoHealth 7, S8-S170.

Odugleh-Kolev, A., 2011, 'Social mobilization and One Health: Collaboration between national, regional and international partners', First International One Health Congress Abstracts, EcoHealth 7, S8-S170. http://dx.doi.org/10.1007/s10393 010-0376-0

Ondzie, A., Reed, P., Sullivan, N., Mulangu, S., Mombouli, J.-V., Rouquet, P. et al., 2011, 'One Health and conservation in practice. Wildlife and human risks of Ebola: The role of conservation organizations in disease surveillance', Presentation at the OIE Global Conference on Wildlife, Paris, 23-25 February.

Reed, P. \& Cameron, K., 2011, 'The role in-situ conservation organizations play in disease surveillance programs', First International One Health Congress Abstracts,
EcoHealth 7, S8-S170. http://dx.doi.org/10.1007/s10393-010-0376-0

Riley, T.V., 2011, 'Clostridium difficile in animals - a significant risk to humans!', First International One Health Congress Abstracts, EcoHealth 7, S8-S170. http://dx.doi. org/10.1007/s10393-010-0376-0

Shears, P., 2000, 'Communicable disease surveillance with limited resources: the scope to link human and veterinary programmes', Acta Tropica 76, 3-7. http:// dx.doi.org/10.1016/S0001-706X(00)00081-4

Swai, E.S., Schoonman, L. \& Darbon, C.J., 2010, 'Knowledge and attitude towards zoonoses among animal health workers and livestock keepers in Arusha and
Tanga, Tanzania', Tanzania Journal of Health Research 12, 1-8. http://dx.doi. org/10.4314/thrb.v12i4.54709, PMid:20737833

Taubenberger, J.K. \& Morens, D.M., 2006, '1918 influenza: The mother of all pandemics', Emerging Infectious Diseases 12, 15-22. PMid:16494711

The United Nations System High Level Task Force (HLTF) on the Global Food Security Crisis and the Updated Comprehensive Framework for Action (UCFA), 1st International One Health Congress Abstracts, EcoHealth 7: S8-S170.

The World Bank, 2010, People, Pathogens and Our Planet. Volume 1: Towards a One Health Approach for Controlling Zoonotic Diseases, viewed n.d., from http:// siteresources.worldbank.org/INTARD/Resources/PPP_Web.pdf

World Health Organisation, 2011, Strong intersectoral partnerships in health: Managing zoonotic public health risks at the human-animal-ecosystem interface, Department of Food Safety and Zoonoses (FOS), World Health Organization, Geneva.

World Health Organisation/Department for International Development- Animal Health Program, 2006, 'The Control of Neglected Zoonotic Diseases: A route to poverty alleviation', Report of a Joint WHO/DFID-AHP Meeting with the participation of FAO and OIE, Geneva, 20 and 21 September 2005.

Woolhouse, M.E.J., 2002, 'Population biology of emerging and re-emerging pathogens', Trends in Microbiology 10, S3-S7.

Woolhouse, M.E.J. \& Gowtage-Sequeria, S., 2005, 'Host range and emerging and reemerging pathogens', Emerging Infectious Diseases 11, 1842-1847. http:// dx.doi.org/10.3201/eid1112.050997, PMid:16485468 